\title{
FACTORES ASOCIADOS A LA FECUNDIDAD ADICIONAL NO DESEADA: ANÁLISIS DE UNA ENCUESTA NACIONAL EN PERÚ
}

\author{
Carolina Mendez-Guerra@1,2,a, Ali Al-kassab-Córdova@1,2,a, Gonzalo Cornejo-Venegas(11,2,a, \\ Andres Quevedo-Ramirez (101,2,a, Kenedy Alva@ ${ }^{3, b}$, Edwin Napanga-Saldaña@i]1,4,c. \\ ${ }^{1}$ Escuela de Medicina, Universidad Peruana de Ciencias Aplicadas, Lima, Perú. \\ ${ }^{2}$ Sociedad Científica de Estudiantes de Medicina de la Universidad Peruana de Ciencias Aplicadas, Lima, Perú \\ ${ }^{3}$ Universidad Complutense de Madrid, Madrid, España. \\ ${ }^{4}$ Centro de Prevención y Control de Enfermedades, Ministerio de Salud, Lima, Perú. \\ a Estudiante de Medicina Humana; b estudiante de doctorado en Economía; ${ }^{c}$ médico epidemiólogo.
}

\section{RESUMEN}

Objetivos: Determinar la asociación entre los factores sociodemográficos y reproductivos con la fecundidad adicional no deseada (FAND) en el Perú. Materiales y métodos: Se realizó un estudio observacional, analítico transversal, a partir de la Encuesta Demográfica y de Salud Familiar (ENDES) 2018 en el Perú. La FAND es una variable que se creó a partir de la diferencia numérica entre la fecundidad obtenida y la fecundidad deseada. La variable FAND, se creó a partir de la diferencia numérica entre la fecundidad obtenida y la fecundidad deseada y se agrupó en dos categorías donde la diferencia numérica positiva correspondió a la presencia de FAND. Se realizó una regresión de Poisson, tanto cruda como ajustada. Resultados: Se analizaron los datos de 6944 mujeres entre 40 y 49 años, con una edad promedio de 44,3 años. Se halló una prevalencia de FAND de 72,5\% (IC95\%: 70,4\%-74,4\%). En el modelo ajustado, se observó que pertenecer al quintil superior de riqueza (RPa 0,80; IC95\%: 0,69-0,93) disminuía la probabilidad de FAND con respecto al quintil intermedio. Por otro lado, provenir del ámbito rural ( $\mathrm{RPa}$ 1,07; IC95\%: 1,01-1,14), aumenta la probabilidad de FAND con respecto al provenir del ámbito urbano. Conclusiones: La prevalencia de FAND en mujeres peruanas de 40 a 49 años que participaron de la ENDES 2018 es alta. Provenir del área rural aumenta la probabilidad de FAND; y pertenecer al quintil superior de riqueza, la disminuye.

Palabras clave: Fecundidad; Perú; Anticonceptivos; Planificación Familiar (Fuente: DeCS BIREME).

Citar como: Mendez-Guerra C, Al-kassab-Córdova A, CornejoVenegas G, Quevedo-Ramirez A, Alva K, Napanga-Saldaña E. Factores asociados a la fecundidad adicional no deseada: análisis de una encuesta nacional en Perú. Rev Peru Med Exp Salud Publica. 2020;37(4):64553. doi: https://doi.org/10.17843/ rpmesp.2020.374.5705.

Correspondencia: Carolina Isabel Méndez Guerra; Jr. Cristóbal de Peralta Sur 1241, dpto. 902, Santiago de Surco, Lima, Perú; mendezguerra.ci@gmaill.com

Recibido: 03/05/2020 Aprobado: $16 / 07 / 2020$ En línea: $11 / 11 / 2020$

\section{FACTORS ASSOCIATED WITH UNWANTED ADDITIONAL FERTILITY: ANALYSIS OF A NATIONAL SURVEY IN PERU}

\section{ABSTRACT}

Objectives: To determine the association between sociodemographic and reproductive factors with unwanted additional fertility (UAF) in Peru. Materials and methods: We carried out an observational, cross-sectional analytical study based on the 2018 Demographic and Family Health Survey (DHS) in Peru. The UAF variable was defined as the numerical difference between the general fertility rate and the total fertility rate. This variable was divided into 2 categories, UAF was considered when the numerical difference was positive. The Poisson regression was used, both crude and adjusted. Results: We analyzed data from 6,944 women with an average age of 44.3 years (range, 40 to 49 ). The prevalence of UAF was found to be $72.5 \%$ (95\% CI: 70.4\%-74.4\%). In the adjusted model, patients in the top wealth quintile (aPR 0.80; 95\% CI: 0.69-0.93) were found to have a lower probability of having unwanted pregnancies when compared to those in the middle quintile. On the other hand, patients from rural areas (aPR 1.07; 95\% CI: 1.01-1.14) had a higher probability of having unwanted pregnancies when compared to those from urban areas. Conclusions: The prevalence of UAF in Peruvian women between 40 and 49 years old who participated in the 2018 DHS is high. Patients from rural areas have a higher probability of having unwanted pregnancies, and those in the top wealth quintile have a lower probability.

Keywords: Fertility; Peru; Contraceptive Agents; Family Planning Policy (Source: MeSH NLM). 


\section{INTRODUCCIÓN}

La fecundidad es una variable demográfica que permite analizar la tendencia de crecimiento de una población ${ }^{(1)}$. En las últimas décadas, esta ha disminuido considerablemente en todas las regiones del mundo ${ }^{(2)}$. América Latina es una de las regiones donde la caída ha sido mayor ${ }^{(2)}$, en el Perú, entre 1986 y 2017 , la tasa de fecundidad disminuyó en $44 \%{ }^{(3)}$. A pesar de esta disminución, se sigue observando una brecha entre la fecundidad deseada y la observada, dicha diferencia se describe como la fecundidad adicional no deseada (FAND) ${ }^{(4)}$. Dicho indicador varía entre los países desarrollados y los países en vías de desarrollo, así como entre la población urbana y rural. En los países desarrollados, la fecundidad deseada es superior a la observada; mientras que en los países en vías de desarrollo, se observa una relación inversa ${ }^{(5,6)}$.

Entre los factores asociados a la FAND podemos encontrar la planificación familiar ${ }^{(7)}$. A pesar de que esta medida ha demostrado ser efectiva en el control del crecimiento poblacional, se ha observado que solo un $18 \%$ de las personas conversan con su médico sobre este tema y que la falta de información es similar en ambos géneros ${ }^{(8)}$. Asimismo, el uso de métodos anticonceptivos modernos no ha variado significativamente, y en las zonas rurales siguen predominando los métodos anticonceptivos tradicionales o folclóricos ${ }^{(3,9)}$.

De la misma forma, la educación de la mujer es un factor importante ${ }^{(10)}$. Amaranta et al. reporta que a un menor nivel educativo la fecundidad observada es superior a la deseada ${ }^{(6)}$. Con respecto al empleo, un estudio retrospectivo realizado en los Estados Unidos, entre 1976 y 2010, afirma que, por cada incremento de 1 punto porcentual en la tasa de desempleo, la tasa de fecundidad se reduce en 0,5 concepciones por cada 1000 mujeres $^{(11)}$.

La violencia sexual también es un factor importante para tomar en consideración. Un estudio realizado en mujeres menores de 53 años víctimas de abuso sexual reportó una mayor frecuencia de abortos e infertilidad ${ }^{(12)}$. Asimismo, se ha observado que la violencia de pareja se asocia a abortos espontáneos, muerte fetal y complicaciones en la salud materna ${ }^{(13)}$.

En suma, la fecundidad es una variable demográfica que repercute, en gran medida, en el desarrollo económico y sociocultural del país. Se postula que la disminución de fecundidad incrementa el nivel educativo de la mujer $y$, con ello, el crecimiento económico ${ }^{(14)}$. A nivel nacional, no se identificaron datos que demuestren una asociación entre los factores previamente descritos y la brecha entre la fecundidad observada y la deseada. Por lo tanto, el objetivo del presente trabajo es determinar los factores sociodemográficos y reproductivos asociados a la FAND en Perú.

\section{MENSAJES CLAVE}

Motivación para realizar el estudio: El presente estudio se realizó con la finalidad de entender mejor las características socioculturales y económicas de la brecha entre la fecundidad deseada y la fecundidad observada en el Perú.

Principales hallazgos: Describimos una fecundidad adicional no deseada (FAND) de 72,5\% a nivel nacional. La FAND es $7 \%$ más probable en el ámbito rural que en el urbano; y $20 \%$ menos probable en el quinto quintil de riqueza.

Implicancias: Nuestro estudio brinda un punto de partida para la formulación de políticas públicas efectivas que incidan sobre los factores estudiados en aquellas regiones con mayores prevalencias de FAND.

\section{MATERIALES Y MÉTODOS}

\section{Diseño del estudio y población de estudio}

Se realizó un estudio observacional de tipo transversal analítico, basado en un análisis secundario de la ENDES 2018 en el Perú. El marco muestral de la ENDES 2018 estuvo constituido por la información estadística y cartográfica actualizada de los Censos Nacionales 2007 y la actualización del Sistema de Focalización de Hogares 2012-2013. El muestreo utilizado por la ENDES fue bietápico, probabilístico de tipo equilibrado, estratificado e independiente, por departamento, por área urbana y rural. Las unidades de muestreo estuvieron constituidas por el conglomerado para vivienda particular en el área urbana, y el área de empadronamiento rural y vivienda particular en el área rural. El tamaño muestral de la ENDES 2018 fue de 36760 viviendas, donde se encuestaron a 34996 mujeres de 15 a 49 años. Nuestro estudio incluyó únicamente a las mujeres de 40 a 49 años, ya que se ha descrito que la probabilidad de gestación se reduce considerablemente al alcanzar los 40 años ${ }^{(15)}$, lo cual se evidencia por un descenso de la tasa de fecundidad en mujeres peruanas a partir de dicha edad ${ }^{(16)}$. Asimismo, se excluyeron aquellos registros que no contenían todas las variables de interés.

\section{Variables}

La variable dependiente se creó a partir de la diferencia numérica entre la fecundidad obtenida y la fecundidad deseada. La fecundidad obtenida se calculó por medio de la suma de los hijos vivos y muertos de las mujeres encuestadas. Para esto se les preguntó: «¿Cuántos hijos vivos tiene?», «¿Cuál es el número de hijos que han muerto?» y «¿Cuál es el número de hijas que han muerto?». Mientras que la fecundi- 
dad deseada se obtuvo con la pregunta: «¿Cuál es su número ideal de hijos de ambos sexos?». Se categorizó la variable FAND donde la diferencia numérica positiva correspondió a su presencia y la diferencia numérica negativa o nula correspondió a su ausencia.

Las variables independientes fueron de carácter sociodemográfico y reproductivo. Entre las variables de carácter sociodemográfico destacaron la edad, la región, el nivel socioeconómico, el estado marital, el grado de instrucción alcanzado por la mujer y su pareja, la ocupación de la mujer y la cobertura del seguro de salud. Por otra parte, las variables del ámbito reproductivo fueron las siguientes: edad cuando se casó por primera vez, edad en la que tuvo su primera relación sexual, edad cuando tuvo su primer parto, deseo de la pareja de tener más hijos, uso de métodos anticonceptivos en algún momento, decisor para el uso de métodos anticonceptivos y decisor final en temas de salud.

\section{Recolección y procesamiento de información}

La ENDES es una encuesta nacional anual, de carácter continuo, realizada por el Instituto Nacional de Estadística e Informática (INEI). Contiene información actualizada sobre la salud reproductiva y salud materno-infantil, por lo cual, permite analizar tendencias y cambios relacionados con dichos tópicos. La base de datos se obtuvo en la sección «Microdatos» del portal web del INEI, disponible en el siguiente enlace: http://iinei.inei.gob.pe/microdatos/. Inicialmente, se seleccionaron las variables de interés descritas previamente, las cuales se encontraron en distintos módulos: (datos básicos, oria de nacimiento - tabla de conocimiento de método), 69 (embarazo, parto, puerperio y lactancia), 70 (inmunización y salud). Seguidamente, la unificación de la base de datos se realizó en el programa RStudio versión 3.5.2 (RStudio Team [2015]. RStudio: Integrated Development for R. RStudio, Inc., Boston, MA) a través de la función «Merge». Finalmente, la base de datos se exportó en formato .dta para su posterior análisis.

\section{Análisis de los datos}

Se analizaron los datos en el programa Stata versión 14.0 (Stata Corporation, College Station, TX, EUA). Se utilizó el módulo de comandos svy para encuestas complejas, con el que se especificaron los ajustes por conglomerado, estrato y factores de ponderación para la descripción de las prevalencias, el análisis bivariado y de regresión múltiple. Se describieron las frecuencias relativas de las variables sociodemográficas y reproductivas. Asimismo, se elaboró un mapa coroplético de Perú para representar gráficamente la distribución de la FAND por departamento, según cuartiles. Luego, se procedió a realizar el análisis bivariado con el test de chi cuadrado entre las variables independientes y la FAND. Por último, se calcularon las razones de prevalencia cruda $(\mathrm{RPc})$ y ajustada $(\mathrm{RPa})$ usando modelos lineales gen- eralizados de familia Poisson con enlace log. Las variables que ingresaron al modelo ajustado se seleccionaron a través del método Stepwise. Se consideró la significancia estadística con un $\mathrm{p}<0,05$.

\section{RESULTADOS}

En la ENDES 2018 participaron 34996 mujeres entre 15 y 49 años. De estas, se excluyó a 28052 menores de 40 años, por considerarse que todavía no habían satisfecho su fecundidad deseada. De esta forma, se incluyó a 6944 mujeres entre 40 y 49 años, con una edad promedio de 44,3 años (DE: 2,51). El $60 \%$ vivió en la costa; el $82,4 \%$ vivió en el área urbana; el $90 \%$ tuvo una pareja estable; el 70,9\% alcanzó al menos el nivel de educación secundario; y el 37,2\% se desempeñó como profesional o en el área de servicios. El 45,1\% contó con una pareja con educación secundaria; y el 33,9\%, con una pareja con educación superior. Solo el $24 \%$ contó con seguro de salud (Tabla 1). El 30\% se casó antes de los 19 años. El 62,5\% refirió que ambos miembros de la pareja deseaban el mismo número de hijos (Tabla 2).

La prevalencia de la FAND fue del 72,5\% (IC95\%: 70,4\%$74,4 \%)$. La FAND fue mayor en Huancavelica (88\%), Cusco $(86,5 \%)$ y Madre de Dios (84,9\%); y menor en Ica $(64,1 \%)$, Callao (65\%) y Tumbes (66,5\%) (Figura 1). Del grupo de mujeres encuestadas, el 60,4\% vivió en la región costa y el $79,6 \%$ en el ámbito urbano; el 93,7\% tuvo una pareja estable; el 66,9\% alcanzó, al menos, el nivel de educación secundaria; y el 34,9\% se desempeñó como profesional o en el área de servicios. El 47,2\% tuvo una pareja con educación secundaria y el $30,2 \%$ con una pareja con educación superior. Solo el $22,9 \%$ contó con seguro de salud. Se encontraron asociaciones estadísticamente significativas entre la FAND y el ámbito de residencia, el nivel socioeconómico, el estado marital, el grado de educación, la ocupación, el grado de instrucción de la pareja y la cobertura por un seguro de salud (Tabla 1).

El 33,9\% se casó antes de los 19 años. El 61,1\% refirió que ambos miembros de la pareja deseaban el mismo número de hijos. El 89,9\% refirió haber usado métodos anticonceptivos modernos y el 69,8\% refirió que la decisión de tomar métodos anticonceptivos era tomada en conjunto con su pareja.

Se encontraron asociaciones estadísticamente significativas entre la edad al momento del primer matrimonio, la edad cuando tuvieron su primera relación sexual, la edad cuando tuvieron su primer parto, el uso de métodos anticonceptivos y la FAND (Tabla 2).

En el modelo de regresión múltiple se encontró que pertenecer al quintil superior de riqueza disminuye en $20 \%$ la probabilidad de FAND (RPa 0,80; IC95\%: 0,69-0,93) con respecto al quintil intermedio. Por otra parte, provenir del ámbito rural aumenta en 7\% la probabilidad de FAND (RPa 1,07; IC95\%: 1,01-1,14) con respecto al ámbito urbano. Ade- 
Tabla 1. Prevalencia de fecundidad adicional no deseada según características sociodemográficas en mujeres de 40 a 49 años que participaron en la ENDES 2018, Perú

\begin{tabular}{|c|c|c|c|c|}
\hline Variable & $\begin{array}{c}\text { Población (\%) } \\
\mathrm{n}=6944\end{array}$ & $\begin{array}{c}\text { FAND (\%) } \\
\mathrm{n}=5034\end{array}$ & IC $95 \%$ & Valor de $\mathrm{p}^{\mathrm{a}}$ \\
\hline Edad (años) ${ }^{b}$ & $\begin{array}{c}44,30 \\
(\mathrm{DE} 2,51)\end{array}$ & - & - & \\
\hline \multicolumn{5}{|l|}{ Región } \\
\hline Costa & 60,0 & 60,4 & $58,3-62,4$ & \multirow{3}{*}{0,463} \\
\hline Sierra & 33,2 & 32,6 & $30,7-34,5$ & \\
\hline Selva & 6,8 & 7,1 & $6,4-7,8$ & \\
\hline \multicolumn{5}{|l|}{ Ámbito } \\
\hline Urbano & 82,4 & 79,6 & $78,3-80,9$ & \multirow{2}{*}{$<0,001$} \\
\hline Rural & 17,6 & 20,4 & $19,1-21-7$ & \\
\hline \multicolumn{5}{|l|}{ Quintil de riqueza } \\
\hline Quintil inferior & 15,9 & 18,5 & $17,2-19,9$ & \multirow{5}{*}{$<0,001$} \\
\hline Segundo quintil & 19,1 & 20,1 & $18,3-22,1$ & \\
\hline Quintil intermedio & 20,9 & 21,5 & $19,7-23,6$ & \\
\hline Cuarto quintil & 21,0 & 20,8 & $18,6-23,2$ & \\
\hline Quintil superior & 23,1 & 19,0 & $16,7-21,6$ & \\
\hline \multicolumn{5}{|l|}{ Estado marital } \\
\hline Con pareja estable ${ }^{c}$ & 90,0 & 93,7 & $92,2-95,0$ & \multirow{2}{*}{$<0,001$} \\
\hline Sin pareja estable ${ }^{d}$ & 10,0 & 6,3 & $5,0-7,8$ & \\
\hline \multicolumn{5}{|c|}{ Nivel de educación de la mujer } \\
\hline Sin educación & 3,7 & 4,1 & $3,4-4,9$ & \multirow{4}{*}{$<0,001$} \\
\hline Primaria & 25,4 & 28,9 & $27,0-31,0$ & \\
\hline Secundaria & 35,7 & 36,7 & $34,3-39,2$ & \\
\hline Superior & 35,2 & 30,3 & $27,7-32,9$ & \\
\hline \multicolumn{5}{|l|}{ Ocupación de la mujer } \\
\hline Desempleada & 5,2 & 5,8 & $4,6-7,3$ & \multirow{6}{*}{$<0,001$} \\
\hline Profesional y servicios & 37,2 & 34,9 & $32,3-37,6$ & \\
\hline Trabajo manual & 27,7 & 28,4 & $26,2-30,7$ & \\
\hline Agricultura & 13,7 & 15,6 & $14,4-16,9$ & \\
\hline Hogar y doméstico & 10,1 & 10,7 & $9,1-12,6$ & \\
\hline Clerical & 6,0 & 4,6 & $3,5-5,9$ & \\
\hline \multicolumn{5}{|c|}{ Nivel de educación de la pareja } \\
\hline Sin educación & 1,1 & 1,2 & $0,9-1,7$ & \multirow{4}{*}{$<0,001$} \\
\hline Primaria & 19,8 & 21,4 & $19,7-23,1$ & \\
\hline Secundaria & 45,1 & 47,2 & $44,6-49,9$ & \\
\hline Superior & 33,9 & 30,2 & $27,6-32,8$ & \\
\hline \multicolumn{5}{|l|}{ Cobertura de seguro de salud } \\
\hline Sí & 24,0 & 22,9 & $20,8-25,2$ & \multirow{2}{*}{0,041} \\
\hline No & 76,0 & 77,1 & $74,8-79,2$ & \\
\hline
\end{tabular}

FAND: Fecundidad adicional no deseada

a Prueba de chi cuadrado

${ }^{\mathrm{b}}$ Media (DE)

${ }^{\mathrm{c}}$ Pareja estable: casada, pareja conviviente o no conviviente.

d Sin pareja estable: soltera, divorciada, viuda 


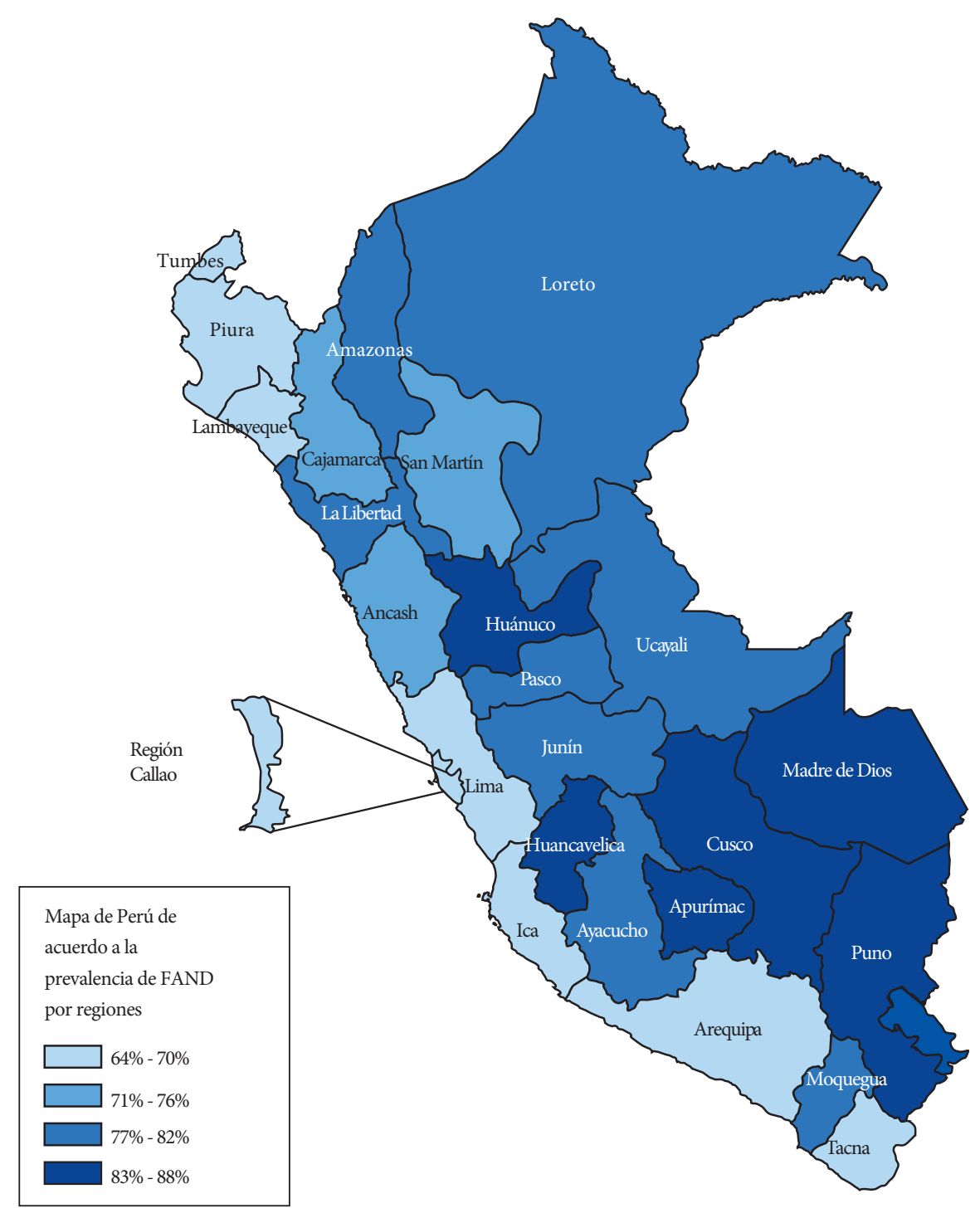

Figura 1. Mapa del Perú de acuerdo con la prevalencia de fecundidad adicional no deseada por regiones.

más, que la entrevistada sea la única decisora sobre sus métodos anticonceptivos aumenta en $10 \%$ la probabilidad de FAND (RPa 1,10; IC95\%: 1,02-1,18) comparada con que la decisión sea tomada en conjunto con su pareja. Igualmente, que la toma de decisiones en materia de salud sea por la entrevistada y un tercero que no sea su pareja, aumenta en 45\% la probabilidad de FAND (RPa 1,45; IC95\%: 1,30-1,62), con respecto a que sea tomada en conjunto con su pareja (Tabla 3).

\section{DISCUSIÓN}

El presente estudio determinó que una alta prevalencia de FAND en mujeres peruanas de 40 a 49 años estuvo presente en siete de cada diez encuestadas. Este hallazgo es particu- larmente importante en aquellos sectores socioeconómicos menos favorecidos. Un estudio nacional halló que en las poblaciones más pobres había una mayor fecundidad y un menor uso de anticonceptivos, a comparación de las poblaciones más adineradas. En el Perú, solo el $34 \%$ de mujeres en edad reproductiva que pertenecen al estrato socioeconómico bajo usan métodos anticonceptivos eficaces ${ }^{(17)}$. De manera similar, un estudio realizado en Bolivia identificó que las mujeres de las minorías étnicas tenían tasas de fecundidad más altas que las mujeres que no pertenecían a dichas minorías, y esta diferencia se atribuyó casi totalmente a la FAND en el grupo de las mujeres de las minorías étnicas ${ }^{(18)}$. Es así como la identificación de los grupos en donde hay mayor FAND es indispensable para reorientar los programas de planificación familiar. 
Tabla 2. Prevalencia de fecundidad adicional no deseada según características reproductivas en mujeres de 40 a 49 años que participaron en la ENDES 2018, Perú

\begin{tabular}{|c|c|c|c|c|}
\hline Variables & $\begin{array}{c}\text { Población (\%) } \\
n=6944\end{array}$ & $\begin{array}{c}\text { FAND (\%) } \\
n=5034\end{array}$ & IC $95 \%$ & Valor de $\mathrm{p}^{\mathrm{a}}$ \\
\hline \multicolumn{5}{|l|}{ Edad del primer matrimonio } \\
\hline$<19$ años & 30,0 & 33,8 & $31,5-36,2$ & \multirow{2}{*}{$<0,001$} \\
\hline$\geq 19$ años & 70,0 & 66,2 & $63,8-68,5$ & \\
\hline \multicolumn{5}{|l|}{ Edad a la primera relación sexual } \\
\hline No ha tenido & 1,6 & 0,0 & $0,0-0,0$ & \multirow{3}{*}{$<0,001$} \\
\hline$<19$ años & 94,1 & 96,89 & $96,0-97,5$ & \\
\hline$\geq 19$ años & 4,2 & 3,1 & $2,5-4,0$ & \\
\hline \multicolumn{5}{|l|}{ Edad al primer parto } \\
\hline$<19$ años & 24,4 & 27,1 & $25,1-29,1$ & \multirow{2}{*}{$<0,001$} \\
\hline$\geq 19$ años & 75,6 & 72,9 & $70,9-74,9$ & \\
\hline \multicolumn{5}{|l|}{ Deseo de la pareja de tener hijos } \\
\hline Mismo número ambos & 62,5 & 61,1 & $57,8-64,2$ & \multirow{4}{*}{0,073} \\
\hline Quiere menos & 9,9 & 9,6 & $7,8-11,4$ & \\
\hline Quiere más & 21,6 & 23,4 & $20,8-26,3$ & \\
\hline No sabe & 6,0 & 6,0 & $4,8-7,6$ & \\
\hline \multicolumn{5}{|l|}{ Uso de métodos anticonceptivos } \\
\hline Nunca usó & 5,9 & 2,8 & $2,1-3,6$ & \multirow{4}{*}{$<0,001$} \\
\hline Método moderno & 87,0 & 89,9 & $88,6-91,1$ & \\
\hline Método folclórico & 0,3 & 0,4 & $0,3-0,6$ & \\
\hline Método tradicional & 6,8 & 6,9 & $5,9-8,1$ & \\
\hline \multicolumn{5}{|c|}{ Decisor sobre el uso de métodos anticonceptivos } \\
\hline En conjunto & 70,8 & 69,8 & $66,1-72,8$ & \multirow{3}{*}{0,143} \\
\hline Ella misma & 24,5 & 26,2 & $23,0-29,4$ & \\
\hline Su pareja & 4,7 & 4,1 & $3,1-5,4$ & \\
\hline \multicolumn{5}{|l|}{ Decisor final en temas de salud } \\
\hline Ambos & 20,3 & 20,3 & $18,2-22,5$ & \multirow{5}{*}{0,332} \\
\hline Entrevistada & 68,3 & 67,7 & $65,2-70,2$ & \\
\hline Entrevistada y alguien más & 0,1 & 0,1 & $0,02-0,3$ & \\
\hline Esposo & 11,2 & 11,8 & $10,4-13,3$ & \\
\hline Alguien más & 0,1 & 0,1 & $0,1-0,3$ & \\
\hline
\end{tabular}

FAND: Fecundidad adicional no deseada

a Prueba de chi cuadrado

De la misma manera, la brecha entre fecundidad deseada y observada, medida en nuestro estudio como FAND, constituye un indicador de insatisfacción del ideal reproductivo ${ }^{(19)}$. En tal sentido, se ha postulado que las medidas de sensibilización orientadas a la reducción del tamaño familiar deseado son necesarias mas no suficientes para alcanzar una reducción significativa de la fecundidad observada. Para ello se requiere expandir el sistema de servicios de control reproductivo, sobre todo en aquellas regiones vulnerables, previa concientización acerca de la importancia de la reducción de la fecundidad deseada ${ }^{(20,21)}$. En tal sentido, nuestro estudio identificó las regiones de Huancavelica, Puno y Cusco como aquellas donde hay mayor FAND.
Asimismo, nuestro estudio halló que el quintil superior de riqueza tiene $20 \%$ menos posibilidades de tener más hijos de los deseados que el quintil intermedio. Un estudio que evaluó factores asociados a la disminución de fecundidad en mujeres peruanas trabajadoras halló que, tanto trabajadoras dependientes como independientes, con un nivel de riqueza alto tienen $60 \%$ menos posibilidades de tener hijos ${ }^{(22)}$. Las diferencias en las cifras citadas pueden ser explicadas por las distintas poblaciones estudiadas. Nuestro estudio analizó mujeres de 40 a 49 años, mientras que el estudio citado analizó mujeres trabajadoras en edad fértil. Otra diferencia es la selección de la variable dependiente puesto que, en el estudio citado, esta fue la probabilidad de tener hijos. No obstante, 
Tabla 3. Análisis de regresión crudo y ajustado para tener fecundidad adicional no deseada en mujeres de 40 a 49 años que participaron de la ENDES 2018, Perú

\begin{tabular}{|c|c|c|c|c|c|c|}
\hline Variable & RP crudo & Valor $\mathbf{p}$ & IC95\% & RP ajustado & Valor de p & IC95\% \\
\hline Edad & 1,00 & 0,942 & $0,99-1,01$ & 0,99 & 0,596 & $0,98-1,00$ \\
\hline \multicolumn{7}{|l|}{ Ámbito } \\
\hline Urbano & Ref & Ref & Ref & Ref & Ref & Ref \\
\hline Rural & 1,22 & $<0,001$ & $0,67-0,72$ & 1,07 & 0,027 & $1,01-1,14$ \\
\hline \multicolumn{7}{|l|}{ Región } \\
\hline Costa & Ref & Ref & Ref & Ref & Ref & Ref \\
\hline Sierra & 0,98 & 0,452 & $0,93-1,03$ & 0,94 & 0,086 & $0,88-1,00$ \\
\hline Selva & 1,02 & 0,455 & $0,96-1,09$ & 0,95 & 0,185 & $0,88-1,02$ \\
\hline \multicolumn{7}{|l|}{ Quintil de riqueza } \\
\hline Quintil inferior & 1,15 & $<0,001$ & $1,08-1,21$ & 1,04 & 0,295 & $0,96-1,13$ \\
\hline Segundo quintil & 1,03 & 0,438 & $0,95-1,10$ & 1,01 & 0,812 & $0,92-1,10$ \\
\hline Quintil intermedio & Ref & Ref & Ref & Ref & Ref & Ref \\
\hline Cuarto quintil & 0,96 & 0,374 & $0,89-1,04$ & 1,01 & 0,724 & $0,92-1,11$ \\
\hline Quintil superior & 0,80 & $<0,001$ & $0,71-0,89$ & 0,80 & 0,005 & $0,69-0,93$ \\
\hline \multicolumn{7}{|l|}{ Nivel de educación de la pareja } \\
\hline Sin educación & Ref & Ref & Ref & Ref & Ref & Ref \\
\hline Primaria & 0,96 & 0,617 & $0,82-1,12$ & 1.01 & 0,870 & $0,87-1,17$ \\
\hline Secundaria & 0,92 & 0,295 & $0,79-1,07$ & 0,99 & 0,951 & $0,85-1,15$ \\
\hline Superior & 0,78 & 0,003 & $0,66-0,92$ & 0,85 & 0,094 & $0,72-1,03$ \\
\hline \multicolumn{7}{|l|}{ Ocupación de la mujer } \\
\hline Desempleada & Ref & Ref & Ref & Ref & Ref & Ref \\
\hline Profesional y servicios & 0,84 & 0,001 & $0,75-0,93$ & 0,92 & 0,111 & $0,82-1,03$ \\
\hline Trabajo manual & 0,91 & 0,104 & $0,83-1,02$ & 0,88 & 0,029 & $0,78-0,99$ \\
\hline Agricultura & 1,03 & 0,580 & $0,93-1,13$ & 0,88 & 0,021 & $0,78-0,98$ \\
\hline Hogar y doméstico & 0,95 & 0,425 & $0,84-1,07$ & 0,91 & 0,273 & $0,79-1,06$ \\
\hline Clerical & 0,68 & 0,001 & $0,54-0,84$ & 0,74 & 0,049 & $0,55-0,99$ \\
\hline \multicolumn{7}{|l|}{ Uso de métodos anticonceptivos } \\
\hline Nunca usó & Ref & Ref & Ref & -- & -- & -- \\
\hline Uso de método moderno & 2,16 & $<0,001$ & $1,74-2,68$ & -- & -- & -- \\
\hline Uso solo folclórico & 2,61 & $<0,001$ & $2,06-3,31$ & -- & -- & -- \\
\hline Uso solo método tradicional & 2,14 & $<0,001$ & $1,70-2,70$ & -- & -- & -- \\
\hline \multicolumn{7}{|c|}{ Decisor sobre uso de métodos anticonceptivos } \\
\hline Decisión conjunta & Ref & Ref & Ref & Ref & Ref & Ref \\
\hline Entrevistada & 1,08 & 0,040 & $1,00-1,16$ & 1,10 & 0,013 & $1,02-1,18$ \\
\hline Esposo & 0,87 & 0,419 & $0,63-1,20$ & 0,89 & 0,386 & $0,68-1,15$ \\
\hline \multicolumn{7}{|l|}{ Decisor final en temas de salud } \\
\hline Ambos & Ref & Ref & Ref & Ref & Ref & Ref \\
\hline Entrevistada & 0,99 & 0,809 & $0,91-1,07$ & 0,99 & 0,954 & $0,92-1,07$ \\
\hline Entrevistada y alguien más & 1,32 & $<0,001$ & $1,23-1,41$ & 1,45 & $<0,001$ & $1,30-1,62$ \\
\hline Esposo & 1,06 & 0,201 & $0,96-1,16$ & 1,02 & 0,700 & $0,93-1,11$ \\
\hline Alguien más & 1,17 & 0,094 & $0,97-1,41$ & 1,03 & 0,743 & $0,85-1,25$ \\
\hline
\end{tabular}

RP: Razón de prevalencia, Ref: Categoría de referencia IC95\%: Intervalo de confianza al 95\%. 
un estudio realizado en Brasil en mujeres de 15 a 49 años halló cifras similares a las de nuestro estudio en referencia al nivel de riqueza. Se reportó que el quintil superior de riqueza tiene $26 \%$ menos posibilidades de tener más hijos ${ }^{(23)}$.

Una mayor probabilidad de FAND se encontró en mujeres que trabajan en los rubros manuales y agrícolas. Estos resultados concuerdan con los estudios anteriores realizados en la realidad latinoamericana, que fueron recogidos en una revisión sistemáti$\mathrm{ca}^{(24)} \mathrm{y}$ describen asociaciones negativas o no significativas entre trabajos tradicionales y la fecundidad. El mismo estudio describe también un menor impacto en la fecundidad en los puestos de trabajo independientes que en los dependientes. Por lo tanto, sugerimos explorar la paradójica contradicción entre el menor acceso a servicios de salud y escolaridad en el ámbito rural (donde ocurre la mayor parte del trabajo agrícola, y que, a su vez es, en su mayor parte, independiente) y esta aparente disminución de la fecundidad. Otro estudio realizado en mujeres africanas se enfocó en las diferencias socioculturales y encontró importantes diferencias en cómo el contexto social influye en el impacto del trabajo sobre la fecundidad, de tal forma que, ciertas variables que impactaban positivamente la fecundidad la disminuían en otros contextos, aun cuando ambos estaban ajustados a las mismas variables. En ese estudio, se describió un impacto negativo del trabajo femenino en la fecundidad, en la sociedad togolesa, donde existe un importante sincretismo entre el cristianismo y el animismo, situación similar a la peruana y que coincide con los resultados de nuestro estudio ${ }^{(25,26)}$.

Nuestro estudio reportó que si la toma de decisiones sobre la salud de la mujer es realizada en conjunto por ella misma y un tercero que no sea su pareja, se incrementa la FAND. Un estudio en Bangladés describió que cuando las decisiones en casa eran tomadas por terceros que no fueran la mujer, la pareja o ambos en conjunto, la probabilidad de usar métodos anticonceptivos disminuía hasta en $40 \%$. Asimismo, se describió una asociación entre la discordancia entre las respuestas de ambos miembros de la pareja y el uso de métodos anticonceptivos por la mujer ${ }^{(27)}$. A fin de explicar este comportamiento, los autores recomendamos profundizar en el estudio de las dinámicas de las familias peruanas.

Con respecto al uso de métodos anticonceptivos, los resultados obtenidos son incongruentes en comparación con un estudio previo que evidencia la planificación familiar como factor protector para la FAND ${ }^{(28)}$. Los autores atribuimos dicha incongruencia a que la ENDES pregunta sobre el uso alguna vez en la vida de métodos anticonceptivos, y es posible que estos sean usados al momento de aplicar la encuesta, pero no al momento de concebir. Asimismo, nuestro estudio encontró que, si la mujer es la única decisora sobre sus métodos anticoncep- tivos, aumenta la probabilidad de FAND. En la revisión de la literatura, no se ha hallado información acerca de la asociación entre la identidad de quién elige el método anticonceptivo y la fecundidad. Sin embargo, se ha descrito que, a pesar del aumento en la frecuencia de uso de métodos anticonceptivos en el Perú, especialmente el condón masculino, la fecundidad no se ha impactado como se esperaría ${ }^{(29)}$. Los autores atribuimos esto al incorrecto o inconstante uso de los métodos anticonceptivos entre las mujeres que los usan.

Una limitación importante de nuestro estudio es que la FAND refleja resultados extrapolables únicamente a poblaciones de características similares a la peruana. Asimismo, la FAND es el resultado de las políticas de planificación familiar de años anteriores, de manera que la FAND en mujeres jóvenes debe evaluarse según los programas de planificación familiar actuales, cuyos resultados se verán en los próximos años. Asimismo, puede existir un sesgo de memoria, pues la ENDES realiza preguntas del estilo «¿alguna vez ha ...?». Además, por tratarse de un estudio transversal, no es posible determinar una relación causal. Por otra parte, entre las fortalezas del presente estudio, destaca que se analizaron variables sociodemográficas y del ámbito reproductivo, tanto de la mujer como de la pareja. Cabe resaltar que el muestreo utilizado por la ENDES 2018 genera que los resultados encontrados puedan ser extrapolables a la realidad que tiene la población peruana femenina de 40-49 años.

La prevalencia de la FAND en las mujeres peruanas de 40 a 49 años que respondieron la ENDES 2018 es alta. Describimos asociaciones significativas con factores sociodemográficos y de carácter reproductivo, así como con las características de la mujer y de las personas de su entorno inmediato. Debido a las limitaciones enfrentadas durante la realización del presente estudio, recomendamos realizar estudios prospectivos que evalúen el impacto de estos factores en la FAND en mujeres desde la coitarquia. Asimismo, sugerimos realizar un estudio cualitativo para evaluar los intereses y opiniones de dicha población.

Contribuciones de los autores: CMG, AAC, GCV, AQR concibieron y diseñaron el artículo, recolectaron los resultados, analizaron e interpretaron datos, redactaron y revisaron el artículo, y aprobaron la versión final del manuscrito. KAC y ENS participaron en la concepción y diseño del artículo, el análisis e interpretación de datos, la revisión crítica del artículo y la aprobación de la versión final. KAC brindó, también, asesoría estadística.

Financiamiento: Autofinanciado.

Conflictos de interés: Los autores declaran no tener conflictos de interés.

\section{REFERENCIAS BIBLIOGRÁFICAS}

1. OMS. Estadísticas Sanitarias Mundiales, 2009 [Internet]. Francia: Observatorio Mundial de la Salud,OMS; 2009 [citado el 24 de abril de 2020]. Disponible en:https://www.who.int/gho/publications/world_health_statistics/2009/es/.
2. Rodríguez-Gómez G, Molina-Cintra MC, Quintana-Llanio L. Fecundidad. Estudio y comportamiento. América Latina y Cuba. Rev Noved Poblac. 2015;11(21):65-77. 
3. INEI. Encuesta Demográfica y de Salud Familiar 2017 [Internet]. INEI 2017 [citado 7 de marzo de 2020]. Disponible en: https://www.inei.gob.pe/ media/MenuRecursivo/publicaciones_digitales/Est/Lib1525/index.html.

4. Gillespie D, Ahmed S, Tsui A, Radloff S. Unwanted fertility among the poor: an inequity? [Internet]. Bull World Health Organ. 2007;85(2):100-7. doi: 10.2471/blt.06.033829.

5. Nishimura T. What are the factors of the gap between desired and actual fertility? - A comparative study of four developed countries [Internet]. School of Economics, Kwansei Gaskuin University. 2012 [citado el 24 de abril de 2020]; 1-34. Disponible en: https://www.researchgate.net/ publication/254426501_What_are_the_factors_of_the_gap_between desired_and_actual_fertility_-_A_comparative_study_of_four_developed_countries.

6. Amarante V, Cabella W. La brecha entre la fecundidad deseada y la observada en Montevideo y su Área Metropolitana. Notas Poblac CEPAL [Internet]. 2015 [citado 7 de marzo de 2020];42. Disponible en: https:// www.cepal.org/es/publicaciones/38521-la-brecha-la-fecundidad-deseada-la-observada-montevideo-su-area-metropolitana.

7. Ministerio de Salud. Norma Técnica de Salud de Planificación Familiar, 2017 [Internet]. Lima: Dirección General de Intervenciones Estrategias en Salud Pública, MINSA; 2017 [citado el 24 de abril de 2020]. Disponible en: http://bvs.minsa.gob.pe/local/MINSA/4191.pdf.

8. Almeida-Santos T, Melo C, Macedo A, Moura-Ramos M. Are women and men well informed about fertility? Childbearing intentions, fertility knowledge and information-gathering sources in Portugal. Reprod Health. 2017;14(1):91 doi: 10.1186/s12978-017-0352-z.

9. OMS. Planificación familiar [Internet]. Organización Mundial de la Salud; 2018 [citado 7 de marzo de 2020]. Disponible en: https://www.who.int/es/ news-room/fact-sheets/detail/family-planning-contraception.

10. Kan K, Lee M-J. The Effects of Education on Fertility: Evidence from Taiwan. Econ Inq. 2018;56(1):343-57. doi: 10.1111/ecin.12492.

11. Currie J, Schwandt H. Short- and long-term effects of unemployment on fertility. Proc Natl Acad Sci USA. 2014;111(41):14734-9. doi: 10.1073/ pnas.1408975111.

12. Ryan GL, Mengeling MA, Booth BM, Torner JC, Syrop CH, Sadler AG. Voluntary and involuntary childlessness in female veterans: associations with sexual assault. Fertil Steril. 2014;102(2):539-47. doi: 10.1016/j. fertnstert.2014.04.042

13. Dhar D, McDougal L, Hay K, Atmavilas Y, Silverman J, Triplett D, et al. Associations between intimate partner violence and reproductive and maternal health outcomes in Bihar, India: a cross-sectional study. Reprod Health. 2018;15(1):109. doi: 10.1186/s12978-018-0551-2.

14. Karra M, Canning D, Wilde J. The Effect of Fertility Decline on Economic Growth in Africa: A Macrosimulation Model. Population and Development Review. 2017;43:237-263. doi: 10.1111/padr.12009.

15. George K, Kamath MS. Fertility and age. J Hum Reprod Sci. 2010;3(3):1213. doi: 10.4103/0974-1208.74152.
16. INEI. Encuesta Demográfica y de Salud Familiar 2018 [Internet]. Lima: INEI; 2018 [citado 26 de marzo de 2020]. Disponible en: https://www. inei.gob.pe/media/MenuRecursivo/publicaciones_digitales/Est/Lib1656/ index1.html.

17. Soriano-Moreno DR, Soriano-Moreno AN, Mejia-Bustamante A, Guerrero-Ramirez CA, Toro-Huamanchumo CJ. Factors associated with highly effective contraceptive use among reproductive-age women in Peru: Evidence from a nationwide survey. Eur J Obstet Gynecol Reprod Biol. 2019;245:114-120. doi: 10.1016/j.ejogrb.2019.12.017.

18. McNamee CB. Wanted and Unwanted Fertility in Bolivia: Does Ethnicity Matter: Int Perspect Sex Reprod Health. 2009;35(4):166-75. doi: 10.1363/ ipsrh.35.166.09.

19. Enríquez-Canto Y, Ortiz-Romaní KJ, Ortiz-Montalvo YJ. Efecto de las preferencias de fecundidad en la cantidad de hijos. Comparación entre trabajadoras peruanas. Horiz. Med. 2018;18(3). doi: 10.24265/horizmed.2018.v18n3.07.

20. Muhoza DM, Broekhuis A, Hooimeijer P. Variations in Desired Family Size and Excess Fertility in East Africa. Int J Popul Res. 2014:11. doi: $10.1155 / 2014 / 486079$.

21. Günther I, Harttgen K. Desired Fertility and Number of Children Born Across Time and Space. Demography. 2016;53(1):55-83. doi: 10.1007/ s13524-015-0451-9.

22. Enríquez Canto Y, Ortiz Romaní KJ, Ortiz Montalvo YJ. Efecto de las preferencias de fecundidad en la cantidad de hijos. Comparación entre trabajadoras peruanas. Horiz Méd. 2018;18(3):37-47. doi: 10.24265/ horizmed.2018.v18n3.07.

23. Oviedo Tejada CA, Madeira Triaca L, Katrein da Costa F, Hellwig F. The sociodemographic, behavioral, reproductive, and health factors associated with fertility in Brazil. PLoS ONE [Internet]. 2017;12(2). doi: 10.1371/ journal.pone.0171888j.

24. Schockaert I. Travail féminin et fécondité en Amériquelatine. Population. 2005;60(1):157-78. doi: 10.3917/popu.501.0157.

25. Beguy D. The impact of female employment on fertility in Dakar (Senegal) and Lomé (Togo). Demogr Res Population. 2009;20(7):97-128. doi: 10.4054/DemRes.2009.20.7.

26. Guerreira MCB. Evangelización y sincretismo religioso en los Andes. Rev Complut Hist América. 1993;19:11-19.

27. Uddin J, Hossin MZ, Pulok MH. Couple's concordance and discordance in household decision-making and married women's use of modern contraceptives in Bangladesh. BMC Women's Health Internet. 2017;17(1):107. doi: 10.1186/s12905-017-0462-3.

28. Barden-O'Fallon JL, Speizer IS, White JS. Association between contraceptive discontinuation and pregnancy intentions in Guatemala. Rev Panam Salud Publica. 2008;23(6):410-7. doi: 10.1590/s1020-49892008000600006.

29. Bellizzi S, Ali MM, Cleland J. Long-Term Trends in Reproductive Behavior Among Young Women in Four Countries, 1995-2009. J Adolesc Health. 2019;64(2):201-10. doi: 10.1016/j.jadohealth.2018.08.014. 